Proc. Indian Acad. Sci. (Earth Planet. Sci.), Vol. 94, No. 1, March 1985, pp. 7-11.

(C) Printed in India.

\title{
Comparison of electron density models with incoherent scatter radar observations at the equator
}

\author{
B T VIKRAM KUMAR and P B RAO \\ Department of Physics, University of Kerala, Kariavattom, Trivandrum 695 581, India \\ MS received 18 May 1984; revised 11 September 1984
}

\begin{abstract}
This paper points out certain discrepancies when the incoherent scatter radar observations at Jicamarca and the electron density models, (the Bent and IRI) were compared. In all cases the bottomside semi-thickness of the observed profile was significantly greater than that of both Bent and IRI models. The decay constants associated with the topside ionosphere for the observed profiles were generally greater than that of the two models, the difference being more pronounced in the IRI model. The results call for a revision of the models so that they accurately represent the electron density distribution at the equator.
\end{abstract}

Keywords. Electron density model; incoherent scatter radar; equator; Bent; International Reference Ionosphere.

\section{Introduction}

Ionospheric models are essential not only for the study of various geophysical problems connected with the upper atmosphere but also for applications like high frequency propagation predictions and evaluation of trans-ionospheric effects on radar and satellite communications. A number of models including the International Reference Ionosphere (IRI) have been developed for many of the above applications (e.g. Nisbet 1971; Ramakrishnan and Rawer 1972; Bent et al 1975; Somayajulu and Ghosh 1979). Some of these provide electron density profiles for any geographic location, local time, season and solar activity. It is important that these models are tested for accuracy by comparing them with observational profiles before they are put to use. This, however, has not been done in a systematic manner for the equatorial latitudes.

In this paper, electron density profiles based on several years' incoherent scatter radar data from Jicamarca $\left(11.95^{\circ} \mathrm{S}, 76.87^{\circ} \mathrm{W}\right)$ were compared with the two widely used models, the Bent and IRI models. The comparison highlights the significant differences between the observational profiles and the empirical models and indicates the need for effecting appropriate corrections.

\section{Description of the models and the observed profiles}

Bent model (Bent et al 1975) is an empirical model derived by using a large number of bottomside and topside soundings as well as satellite-based electron density probe data. The profile is constructed on the basis of the best fit analytical expressions. For this purpose, the entire altitude range below $1000 \mathrm{~km}$ is divided into five segments. The region below the $F_{2^{-}}$peak is represented by a biparabola. The region between the $F_{2^{-}}$ peak and the starting point of the topside exponential portion is represented by a 
parabola. The semithickness parameters of the biparabola and parabola are given as functions of $F_{0} F_{2}$ and the local time. The topside exponential portion is fitted with three segments of differing decay constants which represent the inverse of the corresponding plasma scale heights. The decay constants are given as functions of $F_{0} F_{2}$ and solar flux index. Hence, by feeding in $F_{0} F_{2}, h_{\max }$ and the various constants, the electron density profile can be obtained.

Following the widespread use of atmospheric models like CIRA, a similar effort in the direction of ionospheric modelling was considered worthwhile and the result is the development of an International Reference Ionosphere (Ramakrishnan and Rawer 1972; Rawer 1975). In the IRI model, the general shape of the profile is characterized by a topside exponential portion, a parabolic $F_{2}$-peak region, and another parabolic supplement to represent the $F_{1}$-region. Based on reasonable amount of data, a detailed computer program was developed for which the inputs were the geographic coordinates, sunspot number, month of the year and the time of the day. For these specified conditions various layer parameters were internally calculated and based on these the profile was derived.

The observational profiles were based on the incoherent scatter radar observations at Jicamarca. A total of 207 electron density profiles were collected, stretched over almost a full solar cycle. The profiles were classified into low and high levels of solar activity taking the $F_{107} \mathrm{~cm}$ solar flux of 100 units as the dividing limit. They are further divided into three seasons like summer, winter and equinox. The averaged profiles are obtained for each of the groups for different time periods of the day.

\section{Comparison between the observation and model profiles}

The observational profiles are compared with the Bent and IRI models for the noontime for which the data representation is relatively better than at other times. The corresponding Bent and IRI models for the equator were computed on the basis of the formulations mentioned in $\$ 2$. The Bent model requiring $F_{0} F_{2}$ and $h_{\max }$ as input parameters is normalized naturally at the $F_{2}$-peak with the observational profile. The IRI model, however computes the layer parameters internally and it is found that they differ quite significantly from that of the observational profile. In order to facilitate an easy comparison with the other two, the IRI model is also normalized at the $F_{2}$-peak. Such a modification is also relevant (Rawer 1984). The two models along with the observational profile are shown for different seasons and solar activities in figures 1 and 2. Due to non-availability of data, the comparison could not be done for the summer of high solar activity. The figures show that the observational and model profiles differ significantly. The discrepancies noted among the profiles can be understood by making an approximate fit of the observational and IRI profiles with the Bent model. Using the analytical expressions of Bent model, the decay constants and the thickness parameters are calculated for the corresponding observational and IRI profiles. The Bent parameters extracted for the three profiles given in table 1 show that in all cases, the bottomside semi-thickness of the observational profile is greater than that of both Bent and IRI models. The Bent model assumes equal semi-thickness values for both bottomside and topside portions, for $F_{0} F_{2}<10 \mathrm{MHz}$ which is the case for the observational profiles. But the observational profile shows consistently lower values for the topside portion. The IRI model does not show any consistency in this matter. It can 


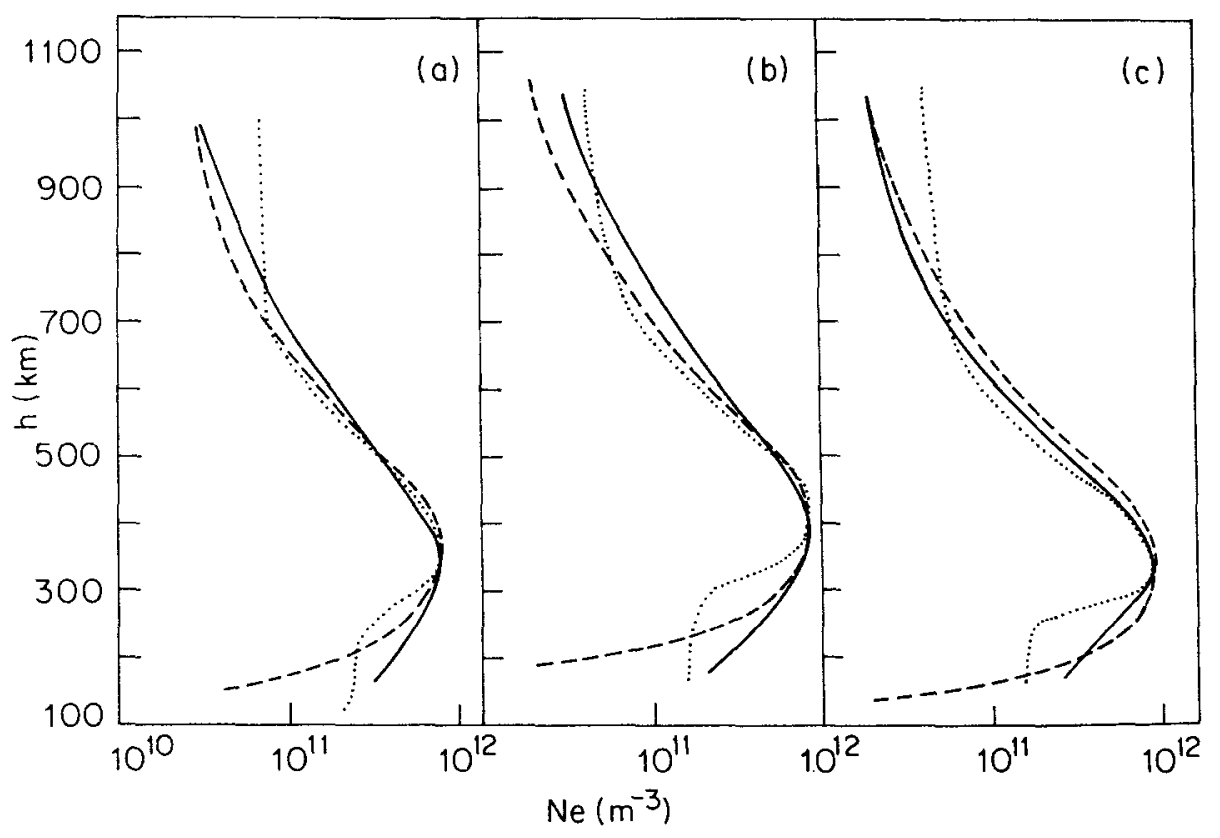

Figure 1. Noon-time profiles of electron density — observational profile, - - Bent model, ..... IRI model for (a) winter (b) equinox (c) summer during low solar activity.

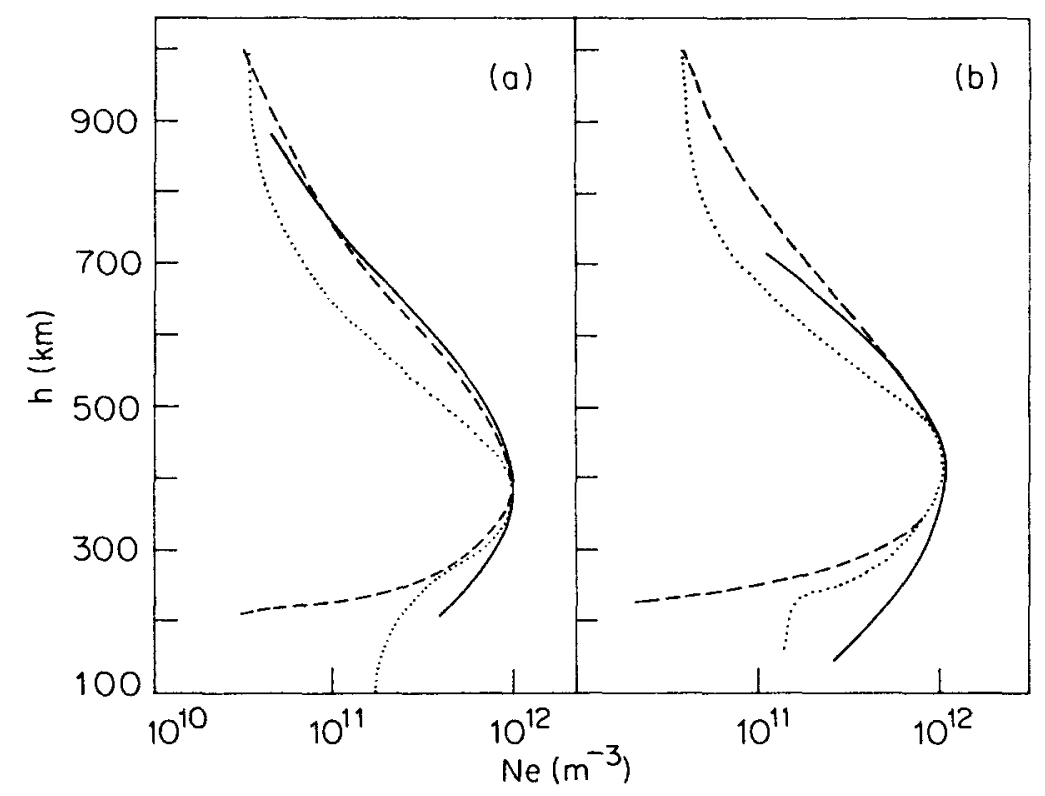

Figure 2. Same as in figure 1 but for (a) winter and (b) equinox during high solar activity. 
Table 1. Bent parameters for observational profile (O), Bent model (B) and IRI model (I).

\begin{tabular}{|c|c|c|c|c|c|c|}
\hline \multirow[b]{2}{*}{ Group } & & \multirow{2}{*}{$\begin{array}{c}\text { Bottomside } \\
\text { semi-thickness } \\
(\mathbf{k m})\end{array}$} & \multirow{2}{*}{$\begin{array}{l}\text { Topside } \\
\text { semi-thick- } \\
\text { ness }(\mathbf{k m})\end{array}$} & \multicolumn{3}{|c|}{$\begin{array}{l}\text { Decay constants of exponential } \\
\text { segments in units of } 10^{-8} / \mathrm{M}\end{array}$} \\
\hline & & & & $K_{1}$ & $K_{2}$ & $\boldsymbol{K}_{\mathbf{3}}$ \\
\hline & $\mathbf{O}$ & 263 & 124 & 6.79 & 6.08 & $3 \cdot 78$ \\
\hline \multirow[t]{3}{*}{ Low-winter } & $\mathbf{B}$ & 233 & 233 & $9 \cdot 45$ & $5 \cdot 55$ & $2 \cdot 70$ \\
\hline & I & 188 & 118 & $8 \cdot 85$ & $7 \cdot 73$ & $1 \cdot 36$ \\
\hline & 0 & 258 & 232 & $7 \cdot 43$ & $7 \cdot 14$ & 3.01 \\
\hline \multirow[t]{3}{*}{ Low-equinox } & $\mathbf{B}$ & 227 & 227 & $9 \cdot 50$ & 5.65 & $2 \cdot 70$ \\
\hline & I & 138 & 177 & 10.71 & $4 \cdot 78$ & $1 \cdot 22$ \\
\hline & $\mathbf{O}$ & 268 & 138 & $10-29$ & $7 \cdot 36$ & 3.01 \\
\hline \multirow[t]{3}{*}{ Low-summer } & B & 215 & 215 & $9 \cdot 60$ & $5 \cdot 80$ & $2 \cdot 75$ \\
\hline & I & 113 & 123 & $11 \cdot 21$ & $4 \cdot 27$ & $1 \cdot 10$ \\
\hline & 0 & 296 & 213 & 7.04 & $7 \cdot 11$ & .. \\
\hline \multirow[t]{3}{*}{ High-winter } & $\mathbf{B}$ & 209 & 209 & 8.00 & $6 \cdot 60$ & 3.65 \\
\hline & I & 239 & 144 & $9 \cdot 80$ & $4 \cdot 32$ & 1.51 \\
\hline & 0 & 325 & 207 & $10 \cdot 24$ & .. & .. \\
\hline \multirow[t]{2}{*}{ High-equinox } & B & 203 & 203 & $8 \cdot 0$ & $6 \cdot 85$ & 3.75 \\
\hline & I & 232 & 134 & 10.20 & 4.64 & 0.90 \\
\hline
\end{tabular}

be seen that for low solar activity, the Bent model is in better agreement with the observational profile than the IRI model as indicated by the bottomside semi-thickness parameters. For high solar activity, however both models seem to differ quite significantly from the observational profile. It is clear that significant modifications are needed in the Bent and IRI model semi-thickness parameters in order that they match the observational profiles around $F_{2}$-peak and below.

If we consider the topside, the decay constants of the first exponential segment for the three profiles are comparable indicating good agreement for the region. The second and third decay constants for the observational profile are generally greater than those for the Bent and IRI models, although the differences noted for the Bent model are considerably smaller as compared to IRI model. The IRI model takes very small values for the third decay constant, which resulted in the pronounced disagreement with the other two models. The small decay constants imply large scale heights which would result normally from the assumption of a lower height level for the heavy-to-light ion transition. It may be that the IRI model is somewhat biased to the mid-and high-latitude regions where the transition levels are known to be comparatively lower than that at equator. It, therefore, seems essential to correct the IRI model for the discrepancy noted above, so that it may fall in agreement with the observational profile.

On the whole it appears that for equatorial latitudes, Bent model agrees better with the observational profiles than the IRI model, presumably because the Bent model is based on a large amount of data from top and bottomside sounders as well as satellitebased electron density probe data with a good latitudinal coverage. On the other hand, IRI model is based on the incoherent scatter radar data, and of the different incoherent scatter radars in the world; only that at Jicamarca is in the equatorial region. It is therefore possible that the IRI model is somewhat biased to the mid-and high-latitude regions. 
The discrepancies noted in the comparison appear to call for a revision in the models, especially IRI, before adopting them to equatorial latitudes. The Bent parameters presented in table 1 clearly suggest the nature of modifications to be effected so that they accurately represent the electron density distribution at the equator.

\section{Acknowledgements}

The authors would like to thank Dr C A Reddy and Dr V V Somayajulu for encouragement and to Dr A P Mitra for providing a computer print-out of the IRI models. Thanks are also due to Dr C V Devasia for assistance. One of the authors (BTVK) would like to thank cSIR for a research fellowship.

\section{References}

Bent R B, Llewellyn S K, Nestercuk G and Schmid P E 1975 in Effect of the ionosphere on space systems and communications (eds) John M Goodman, Naval Research Laboratory, (Washington D C: Naval Res. Lab.) p. 13

Nisbet H 1971 Radio Sci. 6437

Ramakrishnan S and Rawer K 1972 Space Res. 121253

Rawer K 1975 Space Res. 15295

Rawer K 1984 J. Atmos. Terr. Phys. 4691

Somayajulu Y V and Ghosh A B 1979 Indian J. Radio Space Phys. 847 